\title{
Balancing Fertility and Oncologic Outcomes: Can We Have Our Cake and Eat It Too?
}

\author{
Michael Frumovitz \\ Department of Gynecologic Oncology, Anderson Cancer Center, Houston
}

During the last decade, gynecologic oncologists and pathologists have begun to reconsider which clinical and pathologic factors effect outcomes in patients with borderline tumors of the ovary. These tumors of low malignant potential lack the deep stromal invasion $(>5 \mathrm{~mm}$ or $>10 \mathrm{~mm}^{2}$ ) of their invasive carcinoma counterparts. And unlike epithelial carcinomas, which have six different subtypes (serous, endometrioid, mucinous, clear cell, transitional, and undifferentiated), borderline tumors of the ovary are almost exclusively either serous or mucinous. Serous borderline tumors tend to be smaller than their mucinous counterparts and rarely have areas of invasive carcinoma in the same specimen. Furthermore, they seldom recur once removed; however, when they do, they typically return as recurrent borderline histology and rarely undergo malignant transformation into low-grade serous carcinoma. It is exceedingly rare for a serous borderline tumor to recur as a high-grade carcinoma. Mucinous borderline tumors, on the other hand, seem to be a step along the continuum from normal to invasive mucinous carcinoma. Pathologic specimens may have areas of normal ovary, benign mucinous cystadenoma, borderline tumor, and high-grade invasive mucinous carcinoma side by side with one another. Because these neoplasms often can be $>10 \mathrm{~cm}$ in size at surgical resection, making frozen section exclusion of invasive carcinoma difficult, most gynecologic oncologists perform a modified staging procedure, which includes appendectomy for all mucinous neoplasms (benign or borderline) in the event that final pathology returns an invasive carcinoma.

In this month's issue of Annals of Surgical Oncology, Koskas and colleagues ${ }^{1}$ report their single institution

(C) Society of Surgical Oncology 2010

Published Online: 28 October 2010

M. Frumovitz

e-mail: Mfrumovitz@mdanderson.org experience with mucinous borderline tumors of the ovary. This study reviewed a large cohort of 97 patients seen during a 7-year period from 1997-2004. The authors found that $9 \%$ of patients recurred as invasive carcinoma within 5 years of initial therapy. At 10 years follow-up, that number had increased to $13 \%$. This important finding of malignant transformation is significantly higher than what has been reported in the literature to date. Many of these patients had undergone only an ovarian cystectomy in an effort to preserve fertility. The authors challenge the traditional philosophy that borderline tumors of the ovary are relatively benign and that even when they do recur they tend to be indolent with minimal effect on mortality. In addition, they suggest that cystectomy is likely insufficient treatment of borderline tumors and recommend at least complete oophorectomy.

Although retrospective in nature, this study has many strengths. Most importantly, all pathologic specimens were reviewed at their institution. Because $80 \%$ of mucinous tumors of the ovary are extraovarian in origin, it is imperative that these specimens are centrally reviewed to rule out metastatic disease to the ovary. In addition, the authors purposely limited their review to a short time period to minimize practice variability in how these tumors are treated. Due to the rarity of this disease, most published studies on mucinous tumors of the ovary include patients from multiple decades, which inherently introduces great heterogeneity in how these women were treated. Finally, the authors have adequate follow-up because borderline tumors, unlike invasive ovarian carcinomas, often recur remotely from their initial diagnosis. The authors' finding that almost one-third of the recurrences seen in their cohort were after 5 years from diagnosis reinforces the importance of long-term followup of these patients.

Koskas et al. report an excellent overall survival of $96.7 \%$ at 5 years and $91.6 \%$ at 10 years for all women with 
mucinous borderline tumors. They also show disease-free survival of $81.6 \%$ and $77.3 \%$ at 5 and 10 years, respectively. In their multivariate analysis, the only variable associated with an increase of recurrent mucinous borderline tumor of the ovary was the performance of an ovarian cystectomy compared with complete unilateral or bilateral oophorectomy (relative risk 5.6). The authors state that their "results clearly suggest that an oophorectomy should be preferred to a cystectomy to avoid the risk of a (potentially lethal) recurrence in the form of invasive carcinoma." However, in this study there was no statistical difference in recurrence of invasive mucinous carcinoma between the cystectomy and the oophorectomy groups only borderline tumors. Presumably tumor recurrences as carcinoma is what truly effected survival, not recurrent borderline tumors. Other authors have found that salvage in recurrence of borderline tumors (both serous and mucinous) after fertilitysparing surgery is quite high. ${ }^{2}$ Furthermore, they do not report any overall survival data in patients who underwent ovarian cystectomy vs. those who had complete oophorectomy, which makes their conclusion that these recurrences may compromise survival troublesome. In a patient population where the median age is only 36 years, with some patients as young as 12 years as reported in the Koskas article, fertility-sparing surgical approaches are likely to be an important desire for many patients.

As well as recommending oophorectomy over cystectomy, the authors try to clarify the extent of the other surgical procedures that should be performed after a frozen-section diagnosis of mucinous borderline tumor of the ovary. Traditionally, complete surgical staging, including lymph node dissection in addition to appendectomy, has been performed for mucinous borderline tumors. The authors suggest that complete surgical lymph node staging may not be necessary. Lymphadenectomy can likely be bypassed in cases of mucinous borderline tumors, because the rate of lymph node metastasis in clinical stage I invasive carcinoma is exceedingly low. ${ }^{3}$
The authors argue that the use of appendectomy should be considered "optional," which would be a departure from most gynecologic oncologists' current practice. In their 97 patients, 27 had undergone a previous appendectomy before the diagnosis and treatment of the borderline tumor. Of the remaining 70 patients, 23 (33\%) had an appendectomy at the time of their surgery for the borderline tumor and none of these had occult disease in the appendix. However, because $>80 \%$ of mucinous tumors of the ovary are extraovarian in origin, to suggest abandoning appendectomy based on 23 patients is somewhat ambitious, especially when appendectomy is a relatively quick procedure with minimal morbidity. ${ }^{4}$

The article by Koskas et al. is an important addition to the literature about mucinous borderline tumors of the ovary. Their discussion of the best surgical approach is thought-provoking and an excellent debating point for how we balance fertility outcomes with oncologic outcomes. Their finding of a $13 \%$ transformation rate of borderline mucinous tumors into invasive cancers is startling and certainly supports their conclusions that this is not a "safe disease."

\section{REFERENCES}

1. Koskas M, Uzan C, Gouy S, Pautier P, Lhommé C, Haie-Meder C, et al. Prognostic factors of a large retrospective series of mucinous borderline tumors of the ovary (excluding peritoneal pseudomyxoma). Ann Surg Oncol. 2010. doi:10.1245/s10434-010-1293-8.

2. Park JY, Kim DY, Kim JH, Kim YM, Kim YT, Nam JH. Surgical management of borderline ovarian tumors: the role of fertilitysparing surgery. Gynecol Oncol. 2009;113(1):75-82.

3. Schmeler KM, Tao X, Frumovitz M, Deavers MT, Sun CC, Sood $\mathrm{AK}$, et al. Prevalence of lymph node metastasis in primary mucinous carcinoma of the ovary. Obstet Gynecol. 2010; 116(2 Pt 1):269-73.

4. Frumovitz M, Schmeler KM, Malpica A, Sood AK, Gershenson DM. Unmasking the complexities of mucinous ovarian carcinoma. Gynecol Oncol. 2010;117(3):491-6. 